\title{
CREBRF is a potent tumor suppressor of glioblastoma by blocking hypoxia-induced autophagy via the CREB3/ATG5 pathway
}

\author{
HAO XUE ${ }^{1,2^{*}}$, JINSEN ZHANG $^{1,2^{*}}$, XING GUO $^{1,2^{*}}$, JIAN WANG $^{3}$, JIANGBING LI $^{4}$, XIAO GAO $^{1}$, \\ XIAOFAN GUO ${ }^{1}$, TONG LI ${ }^{1}$, SHUGANG XU ${ }^{1,5}$, PING ZHANG ${ }^{2}$, QINGLIN LIU $^{1}$ and GANG LI ${ }^{1,2}$ \\ ${ }^{1}$ Department of Neurosurgery, Qilu Hospital of Shandong University; ${ }^{2}$ Brain Science Research Institute, Shandong University, \\ Jinan, Shandong 250012, P.R. China; ${ }^{3}$ Department of Biomedicine, University of Bergen, 5009 Bergen, Norway; \\ ${ }^{4}$ Department of Cardiology, Shandong Provincial Hospital of Shandong University, Jinan, Shandong 250021; \\ ${ }^{5}$ Department of Neurosurgery, Dezhou People's Hospital, Dezhou, Shandong, P.R. China
}

Received January 29, 2016; Accepted March 10, 2016

DOI: $10.3892 /$ ijo.2016.3576

\begin{abstract}
Hypoxia induces protective autophagy in advanced glioblastoma cells, and targeting this process may improve the outcome for glioblastoma patients. Recent studies have suggested that the autophagic process is upregulated in glioblastoma cells in response to extensive hypoxia. Here, we describe a novel tumor suppressor in glioblastoma cells, whereby hypoxia downregulated CREBRF expression and acts as a potent inhibitor of autophagy in glioblastoma cells via the CREB3/ATG5 pathway. Our results demonstrate that CREBRF expression negatively correlates with autophagic and HIF-1 $\alpha$ levels in different grade gliomas. Given that CREBRF is a negative regulator of CREB3, CREB3 knockdown also repressed hypoxia-induced autophagy in glioblastoma cells in vitro. Collectively, our findings provide new insight into the molecular mechanisms underlying hypoxia-induced glioblastoma cell autophagy and indicate that the hypoxia/CREBRF/ CREB3/ATG5 pathway plays a central role in malignant glioma progression.
\end{abstract}

\section{Introduction}

As the most malignant type of brain tumor, gliomas account for $>70 \%$ of all brain tumors (1), and the most common type of glioma is glioblastoma (GBM) (2). The properties of GBM are generally thought to include high mortality and recurrence rates (3), uncontrollable invasiveness (4), potent angiogenesis (5), a widespread hypoxic region $(6,7)$, and upregulated autophagic processes $(8,9)$. Hypoxia is a common feature in solid tumors

Correspondence to: Dr Gang Li or Dr Qinglin Liu, Department of Neurosurgery, Qilu Hospital of Shandong University, 107 Wenhua Western Road, Jinan, Shandong 250012, P.R. China

E-mail: ligangqiluhospital@163.com

E-mail: bodystrong@126.com

*Contributed equally

Key words: hypoxia, autophagy, glioblastoma, CREBRF, CREB3, ATG5 due to rapid progression and a relatively inadequate blood supply, and tumor hypoxia is an independent prognostic factor associated with poor survival $(6,10,11)$. Numerous studies have suggested that hypoxia activates multiple cellular processes in tumors, such as proliferation (12), angiogenesis (7), migration (13), invasion (12), and recently, autophagy $(8,14)$.

The term autophagy means 'self-eating'. Autophagy is a process that involves the formation of double-membrane autophagosomes containing damaged or old organelles $(15,16)$. Autophagy provides tumor cells with reclaimed essential elements for survival through starvation, hypoxia, immune response and chemoradiotherapy (17). Because tumor cells are over-consumptive, these cells develop ubiquitous autophagy to overcome the relatively infertile tumor microenvironment (18-20). In recent years, accumulating evidence has demonstrated that autophagy favors tumor development (21). Nevertheless, the detailed mechanisms by which hypoxia induces autophagy remain unclear.

CREBRF (CREB3 regulatory factor) is a novel cellular protein and a specific negative regulator of CREB3 that can recruit nuclear CREB3 out of nucleus and promote CREB3 protein degradation (22). CREB3 (also called Luman) is the primary member of the CREB (cyclic-AMP-responsive element binding) family (CREB1, CREB2 and CREB3) (23). CREB family proteins bind to the cAMP-responsive element (CRE) recognition sequence of cAMP-sensitive genes to regulate transcription $(23,24)$. Furthermore, all CREB3 family sub-members appear to play a role in the unfolded protein response (UPR) (22). Although CREB1 can upregulate autophagy genes $(25,26)$ and CREBRF is involved in inducing cell apoptosis through the ER stress pathway (27), there is no evidence that the CREBRF/CREB3 pathway is directly involved in regulating autophagy in tumor cells.

In this study, we report a novel autophagy-promoting mechanism in hypoxic tumor cells in which hypoxia downregulated CREBRF expression and promotes tumor cell autophagy by upregulating CREB3 in GBM cells through the ATG5dependent pathway. We first investigated the key autophagic regulating effect of CREBRF/CREB3 during the hypoxia process. We demonstrate that hypoxic pretreatment decreases CREBRF expression in glioblastoma cells and significantly 
increases CREB3 levels. Importantly, knocking down endogenous CREB3 alleviates hypoxia-induced autophagy. To understand the mechanisms of the autophagy induced by CREB3, we screened most autophagy-related genes (ATGs) using quantitative real-time PCR. Finally, we provide evidence that ATG5 has a central role in the autophagic inducing effect of CREB3. Our results suggest potential uses for anti-CREB therapeutic strategies in adjuvant therapy for glioma patients.

\section{Materials and methods}

Tissue samples and cell lines. The human glioma cell lines T98G and U87 were purchased from the Chinese Academy of Sciences Cell Bank and identified by the STR site detection assay (Microread Genetics Co., Beijing, China). In addition, 76 human glioma tissue samples including 32 low-grade gliomas (4 grade I tumors and 28 grade II tumors), 44 highgrade gliomas (16 grade III tumors and 28 grade IV tumors), and 2 normal brain tissues from decompression operation were obtained from the Department of Neurosurgery of Qilu Hospital of Shandong University from October 2013 to June 2015. The glioma specimens were verified and classified according to the WHO classification standard of tumors by two experienced clinical pathologists. Our study was approved by the Institutional Review Board of Shandong University. Written informed consent was obtained from all the patients, and the hospital ethics committee approved the experiments.

Cell culture and hypoxia treatment. The cells were cultured in DMEM (Invitrogen) supplemented with 10\% FBS (Gibco) and maintained at $37^{\circ} \mathrm{C}$ with $5 \% \mathrm{CO}_{2}$ in a humidified chamber. Hypoxic conditions were induced by incubating the cells in a modular incubator chamber flushed with a gas mixture containing $1 \% \mathrm{O}_{2}, 5 \% \mathrm{CO}_{2}$, and $94 \% \mathrm{~N}_{2}$ at $37^{\circ} \mathrm{C}$.

Immunohistochemical staining. Human glioma tissue samples or solid tumors removed from sacrificed mice were fixed with $4 \%$ formaldehyde. Paraffin-embedded tumor tissues were sectioned to $5-\mu \mathrm{m}$ thickness and mounted on positively charged microscope slides, and $1 \mathrm{mM}$ EDTA ( $\mathrm{pH}$ 8.0) for HIF-1 $\alpha$ or citrate solution ( $\mathrm{pH}$ 6.0) for other antigens was used for antigen retrieval. Endogenous peroxidase activity was quenched by incubating the slides in methanol containing $3 \%$ hydrogen peroxide, followed by washing in PBS for $6 \mathrm{~min}$. The sections were incubated for $2 \mathrm{~h}$ at room temperature with normal goat serum and subsequently incubated at $4^{\circ} \mathrm{C}$ overnight with primary antibodies (Abcam, 1:200 HIF-1 $\alpha, 1: 400$ LC3B, and 1:300 CREB3. Cell Signaling Technology, 1:300 cleaved caspase 3 ). The sections were then rinsed with PBS and incubated with horseradish peroxidase-conjugated goat anti-rabbit or anti-mouse antibodies, followed by reaction with diaminobenzidine and counterstaining with Mayer's hematoxylin. H\&E staining was performed free of charge by the pathology department of Qilu Hospital of Shandong University. Evaluation of the staining reaction was performed in accordance with the immunoreactive score (IRS): IRS = SI (staining intensity) x PP (percentage of positive cells). An SI value of 0 was negative; 1 , weak; 2 , moderate; and 3 , strong. A PP value of 0 was negative; $1,10 \%$ positive cells; $2,11-50 \%$ positive cells; $3,51-80 \%$ positive cells; and $4,>80 \%$ positive cells. Five visual fields from different areas of each tumor were used for the IRS evaluation.

GFP-LC3 stable cell lines and quantitative GFP-LC3 analyses. A T98G GFP-LC3 stable cell line was established by transient transfection of the pSELECT-GFP-LC3 lentiviral vector (Invivogen, USA). GFP-LC3 puncta formation under normoxic or hypoxic conditions was determined by capturing images using a DP71 CCD digital camera microscope (Olympus). To quantify autophagic cells after treatment, we counted the number of autophagic cells as determined by the presence of GFP-LC3 puncta $(\geq 20$ puncta $=$ a positive cell) in 200 cells.

Western blot analysis. After the desired treatment, cells were washed twice with cold PBS and harvested with a rubber scraper. Cell pellets were lysed and kept on ice for $\geq 30 \mathrm{~min}$ in a buffer containing $50 \mathrm{mM}$ Tris- $\mathrm{HCl}$ (pH 7.4), $150 \mathrm{mM}$ $\mathrm{NaCl}, 0.5 \%$ Nonidet P-40, $50 \mathrm{mM} \mathrm{NaF}, 1 \mathrm{mM} \mathrm{Na} \mathrm{VO}_{4}, 1 \mathrm{mM}$ phenylmethylsulfonyl fluoride, and $1 \mathrm{mM}$ PMSF. The lysates were cleared by centrifugation, and the supernatants were collected. Cell lysates were then separated by SDS-PAGE and subjected to western blot analysis with primary antibodies and horseradish peroxidase-conjugated secondary antibodies. Antibodies against the following were used for western blotting: P62 (Cell Signaling Technology, 5114S), LC3B (Abcam, 63817), GAPDH (Cell Signaling Technology, 3683S), caspase 3 (Cell Signaling Technology, 9668T), cleaved caspase 3 (Cell Signaling Technology, 9664S), CREBRF (Santa Cruz Biotechnology, sc-133747), CREB3 (Abcam, ab42454), and ATG5 (Abcam, ab108327). GAPDH served as the loading control in all experiments involving hypoxic treatment due to the upregulation of $\beta$-actin under hypoxia.

Small interfering RNA transfection. CREB3 and negative control siRNAs were synthesized by Rio-Bio (China). The sequences of CREB3 siRNAs were as follows: CREB3-1299, 5'-GCA GUC AGA AGU GCC GAA ATT-3' (sense) and 5'-TTC GUC AGU CUU CAC GGC UUU-3' (antisense). The sequences of negative control were as follows: 5'-UUC UCC GAA CGU GUC ACG UTT-3' (sense) and 5'-ACG UGA CAC GUU CGG AGA ATT-3' (antisense). The siRNAs were transfected into T98G and U87 cells for $48 \mathrm{~h}$ using Lipofectamine 2000 according to the protocol of the manufacturer.

Cell viability assay. The CCK-8 assay (Dojindo, Japan) was used to test cell viability. Tumor cells in medium containing $10 \%$ fetal bovine serum were seeded into 96 -well, flat-bottomed plates at $5 \times 10^{3}$ cells/well and incubated at $37^{\circ} \mathrm{C}$ overnight. After the desired treatment, the cells were incubated for an additional $4 \mathrm{~h}$ with $100 \mu \mathrm{l}$ of serum-free DMEM and $10 \mu \mathrm{l}$ of CCK- 8 at $37^{\circ} \mathrm{C}$. The absorbance at $450 \mathrm{~nm}$ was measured using a microplate reader.

Annexin V-fluorescein isothiocyanate (FITC) assay. To assess the degree of apoptosis of different groups of glioma cells, the extent of Annexin V-FITC/propidium iodide (PI) staining was determined by flow cytometry using the Annexin V/PI staining kit from Bender MedSystems (Vienna, Austria). Samples were 
Table I. Demographic parameters of patients participating in the study.

\begin{tabular}{lcr}
\hline & No. of patients & N \% \\
\hline Assessable & & \\
Glioma & 76 & 97.44 \\
Normal brain tissues & 2 & 2.56 \\
Gender & & \\
Male & 48 & 61.54 \\
Female & 30 & 38.46 \\
Age (years) & & \\
Median (range) & $46.41(6-75)$ & \\
Pathological type & & \\
Astrocytoma & 22 & 28.21 \\
Anaplastic astrocytoma & 12 & 15.38 \\
Pilocytic astrocytoma & 3 & 3.85 \\
Oligodendroglioma & 7 & 8.97 \\
Anaplastic oligodendroglioma & 4 & 5.13 \\
Glioblastoma & 28 & 35.90 \\
Normal brain tissues & 2 & 2.57 \\
WHO tumor grade at diagnosis & & \\
I & 4 & 5.26 \\
II & 28 & 36.84 \\
III & 16 & 21.05 \\
IV & 28 & 36.84 \\
\hline
\end{tabular}

measured using an Epics XL-MCL flow cytometer (Beckman Coulter, Brea, CA, USA) and analyzed with WinMDI 2.8 software.

TUNEL assay. Glioma cells were plated on glass slides in 24-well culture plates at $2 \times 10^{5}$ cells/well for $24 \mathrm{~h}$ and subsequently treated with drugs for an additional $48 \mathrm{~h}$ in serum-free DMEM. Glass slides with glioma cells and paraffin-embedded tumor sections were stained by the TUNEL technique using a TACS $^{\circledR 2}$ TdT-Fluor in situ apoptosis detection kit (Trevigen, Inc.) according to the instructions of the manufacturer. TUNEL-positive cells were counted from $\geq 10$ random fields under a fluorescence microscope.

$R N A$ extraction and real-time quantitative PCR. Total RNA was extracted using TRIzol according to the manufacturer's protocol. Then, total RNA (50 ng) was reverse-transcribed with miR stem-loop RT primers or with U6 RT primers using a ReverTra Ace qPCR RT kit according to the manufacturer's protocol to generate cDNA. Real-time PCR was performed using a SYBR Premix Ex Taq ${ }^{\mathrm{TM}}$ kit with each primer. The reactions were performed using a LightCycler 2.0 Instrument. U6 expression was used as the endogenous control. The absolute expression levels were calculated as concentration ratios using a Roche LightCycler ${ }^{\circledR} 2.0$ system.

Statistical analysis. Data analyses were conducted with SPSS 16.0 (SPSS, IL, USA) and GraphPad-Prism5 (GraphPad,
CA, USA). Descriptive statistics including means \pm SD, Student's t-test, non-parametric Kruskal-Wallis tests for multiple comparison, the Mann-Whitney U test for two-group comparison, Kaplan-Meier plots, log-rank tests, one-way ANOVAs, and Pearson's correlation test were used to analyze significant difference; ${ }^{*} \mathrm{P}<0.05,{ }^{* *} \mathrm{P}<0.01$, and ${ }^{* * * *} \mathrm{P}<0.001$ were considered statistically significant.

\section{Results}

CREBRF/CREB3 levels correlate with human glioma grade. To analyze CREBRF/CREB3 expression in clinical samples, total RNA was extracted from surgically removed glioma tissues of 20 patients ( 2 normal brain tissue samples, 9 patients with WHO I-II and 9 patients with WHO III-IV gliomas), and RT-PCR was performed. Interestingly, significant differences in CREBRF expression were observed between low-grade (I-II) and high-grade (III-IV) patients. CREBRF expression was significantly reduced in high-grade glioma tissues compared with low-grade glioma tissues or normal brain tissues (Fig. 1A, left). Consistent with its negative regulating property, CREB3 expression was significantly reduced in low-grade glioma tissues compared with high-grade glioma tissues (Fig. 1A, right) with a significant negative correlation (Pearson correlation index, $-0.6112 ; \mathrm{P}$-value $=0.0042)$ (Fig. 1B). To further examine whether CREB3 expression correlates with the WHO grades of human glioma, we performed immunohistochemical staining to detect CREB3 expression in 76 human glioma specimens with different grades and 2 normal brain tissues (Table I). As shown in Fig. 1C, CREB3 expression positively correlated with the WHO grade of glioma (Fig. 1D). Furthermore, patients with clinical survival information were analyzed by Kaplan-Meier estimation. We found that glioma patients with high CREB3 expression exhibited significantly poor postoperative survival time (Fig. 1E). The above findings raised the intriguing possibility that CREBRF acts as a tumor suppressor and downstream CREB3 acts as a prognostic biomarker of glioma. The underlying mechanisms by which CREBRF suppresses the malignant progression of glioma remain uncertain.

CREB3 levels correlate with the density of autophagic cells and HIF-1 $\alpha$ levels in human glioma tissues. Extensive autophagy in the hypoxic areas of tumors has been described (14). However, the association among CREB3 and LC3B levels and hypoxia levels in gliomas has not been reported, and the CREB3-mediated upregulation of autophagy in hypoxic areas is also unknown. To examine whether CREB3 expression correlates with autophagy and hypoxia levels in human glioma, we detected HIF-1 $\alpha$ (a marker of hypoxia) and LC3B (a marker of autophagy) expressions in 76 human glioma specimens, which represent different grades and 2 normal brain tissue samples, using immunochemical staining. As shown in Fig. 2A, HIF-1 $\alpha$ and LC3B expression levels strongly correlated with the WHO grade of glioma (Fig. 2B). In addition, HIF- $1 \alpha$, and LC3B expression positively correlated with CREB3 levels in glioma (Fig. 2C). Taken together, these results demonstrate that CREB3 expression correlates with the density of autophagic cells and HIF-1 $\alpha$ levels in gliomas. 

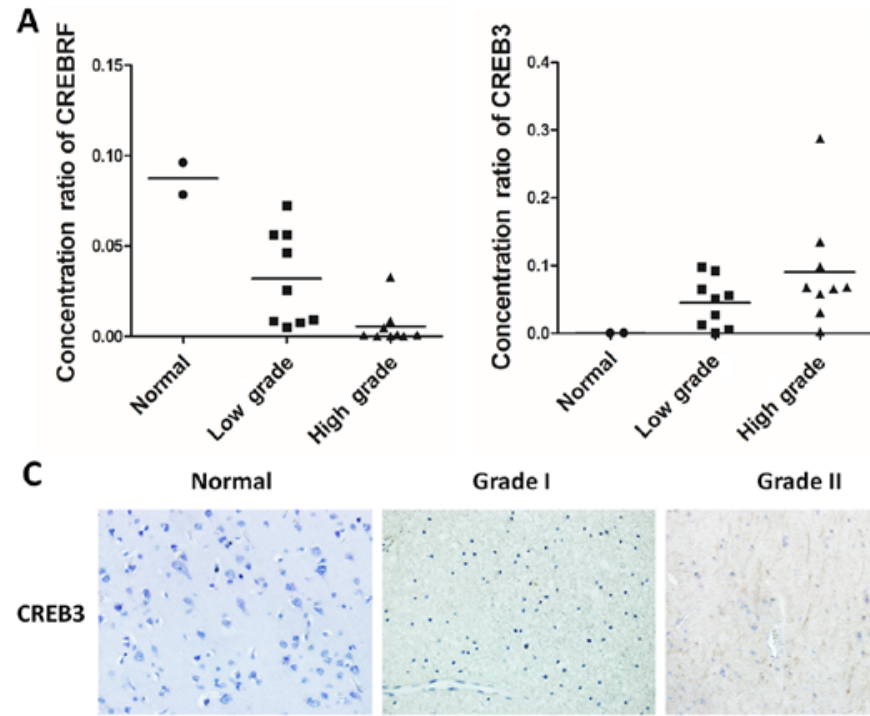

D

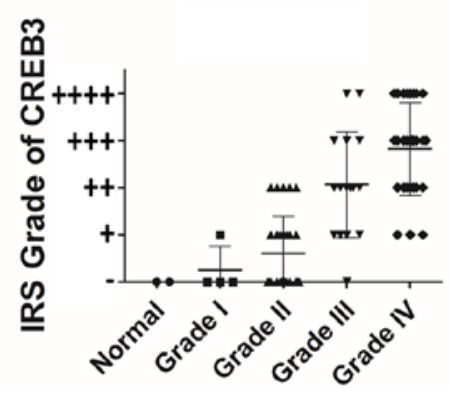

Grade II
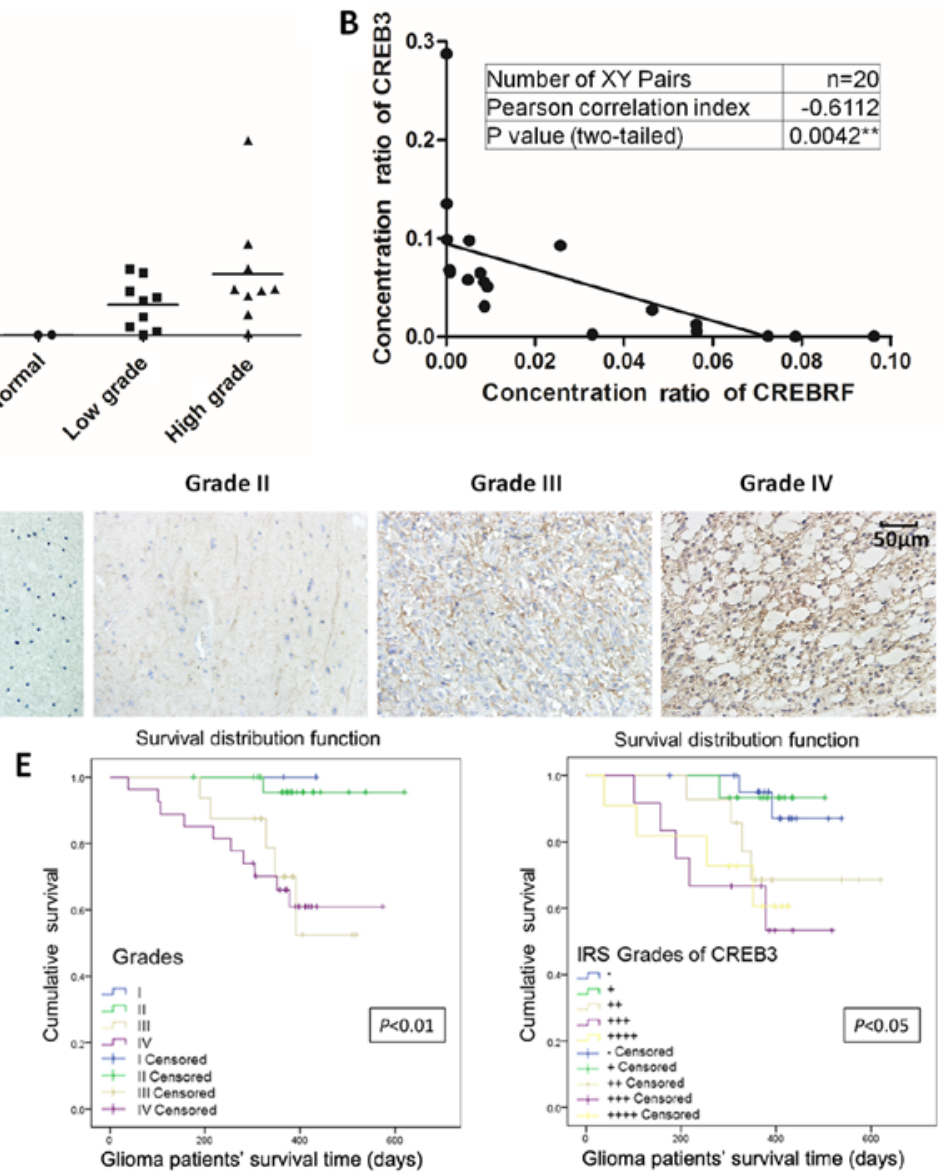

Figure 1. CREBRF/CREB3 levels correlate with the human glioma grade. (A) CREBRF and CREB3 expression levels in clinical samples that were surgically removed from glioma tissues of 20 patients ( 2 normal brain tissue samples, 9 patients with WHO I-II and 9 patients with WHO III-IV gliomas) were assessed by quantitative real-time PCR. ${ }^{* *} \mathrm{P}=0.0028$, Mann-Whitney U test of CREBRF expression in high-grade vs. low-grade gliomas. (B) Pearson's rho rank correlation tests of CREBRF and CREB3 expression levels in clinical samples. (C) CREB3 expression positively correlated with the WHO grade of gliomas. Seventy-six human gliomas representing different WHO grades and two normal brain tissue samples were processed by immunohistochemistry. (D) Quantification of IHC staining in glioma tissues representing different WHO grades. (E) Prognostic significance of CREB3 expression in glioma patients. According to the IRS score of CREB3, Kaplan-Meier survival curves of glioma patients revealed that high CREB3 expression correlated with poor prognosis. The log-rank (Mantel-Cox) significance is $\mathrm{P}=0.035$.

A

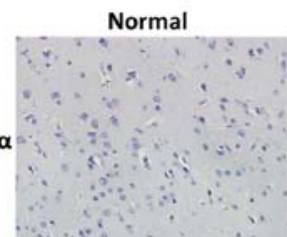

C3B

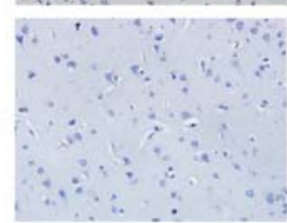

B

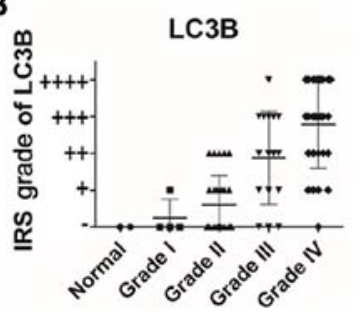

Grade

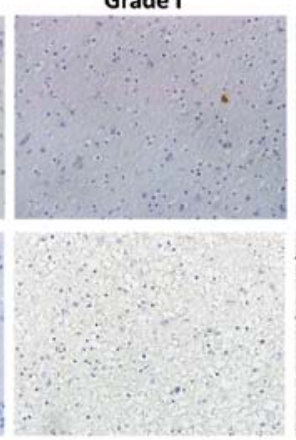

HIF-1a

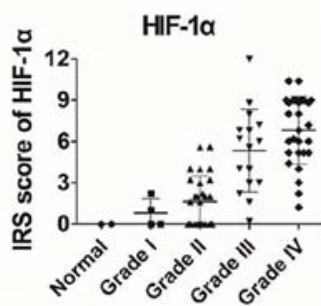

Grade II

Grade III

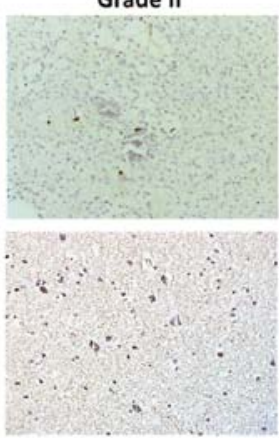

C

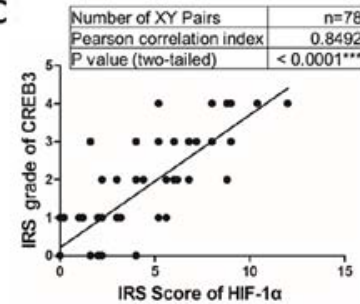

Grade IV
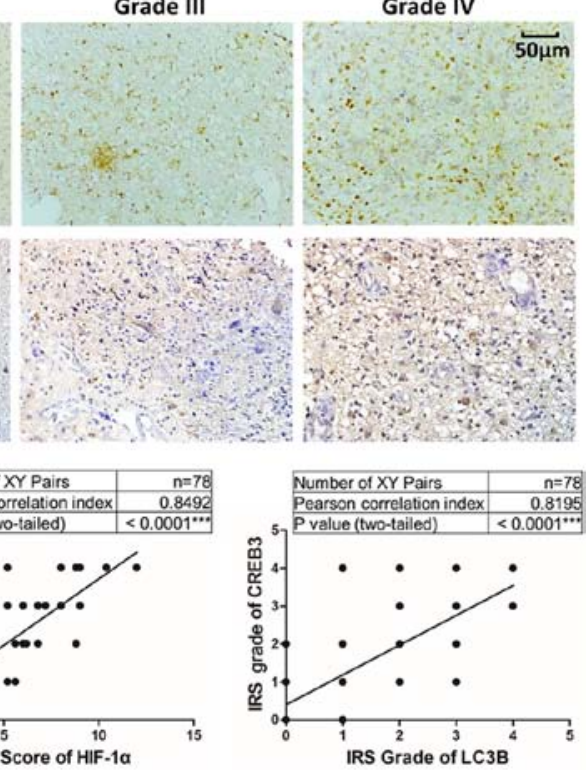

Figure 2. CREB3 levels correlate with the density of autophagic cells and HIF-1 $\alpha$ levels in human glioma tissues. (A) HIF-1 $\alpha$ and LC3B expression positively correlated with the WHO grade of gliomas. CREB3 expression positively correlated with the WHO grade of gliomas. Seventy-six human gliomas representing different WHO grades and two normal brain tissue samples were processed by immunohistochemistry. (B) Quantification of IHC staining of glioma tissues representing different WHO grades. (C) Pearson's rho rank correlation tests of HIF-1 $\alpha$, LC3B and CREB3. 
A
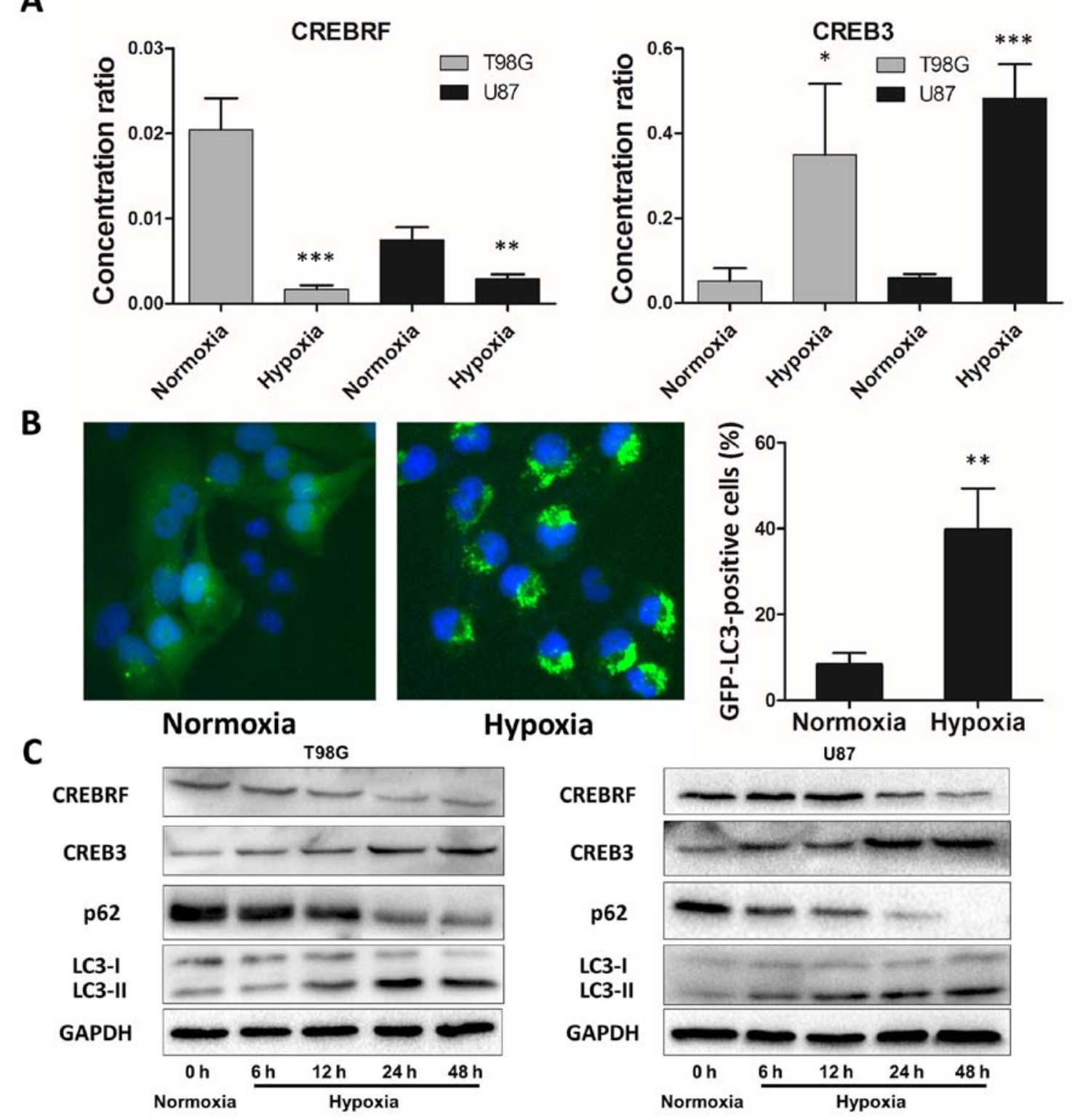

Hypoxia

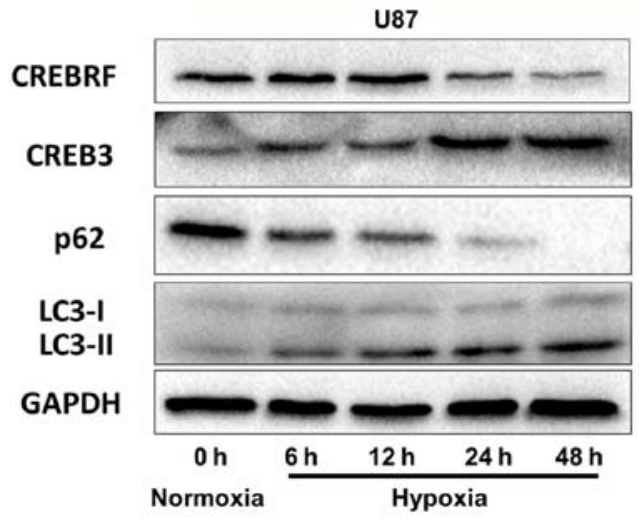

Figure 3. Hypoxia induces autophagy activation and CREB3 upregulation in glioblastoma cells. (A) CREBRF and CREB3 expression levels in hypoxic T98G and U87 cells (hypoxia treatment for $24 \mathrm{~h}$ ) were assessed by quantitative real-time PCR. The data presented are the mean \pm SD of five independent experiments. ${ }^{*} \mathrm{P}<0.05,{ }^{* *} \mathrm{P}<0.01,{ }^{* * *} \mathrm{P}<0.001$, one-way ANOVA. (B) Hypoxia promotes GFP-LC3B translocation. pSELECT-GFP-LC3B transfection revealed LC3B puncta in T98G cells treated with hypoxia $\left(1 \% \mathrm{O}_{2}, 5 \% \mathrm{CO}_{2}\right.$, and $94 \% \mathrm{~N}_{2}$ at $\left.37^{\circ} \mathrm{C}\right)$ for $24 \mathrm{~h}$. Cells were fixed and stained with DAPI for nuclear visualization. Representative images are presented. Quantitative analysis of GFP-LC3B puncta is presented in the right panel. At least 100 cells were examined in each experimental group. The data presented are the mean \pm SD of three independent experiments. ${ }^{* *} \mathrm{P}<0.01$, two-tailed t-test. (C) Hypoxia induced LC3B conversion and SQSTM1 degradation in T98G and U87 cells (hypoxia treatment for 0, 6, 12, 24 and 48 h). CREBRF, CREB3, LC3B and p62 levels were examined by western blot analysis in glioblastoma cells after hypoxia treatment $\left(1 \% \mathrm{O}_{2}, 5 \% \mathrm{CO}_{2}\right.$, and $94 \% \mathrm{~N}_{2}$ at $\left.37^{\circ} \mathrm{C}\right)$. GAPDH served as the loading control.

Hypoxia induces autophagy activation and CREB3 upregulation in glioblastoma cells. We further verified CREBRF and the CREB3 levels in hypoxia T98G and U87 cells using quantitative real-time PCR, and our results are consistent with results from human glioma tissue samples. CREBRF expression significantly decreased in glioblastoma cells after $24 \mathrm{~h}$ of hypoxia treatment, and CREB3 increased accordingly (Fig. 3A). It is generally accepted that hypoxia activates autophagy as an evolutionarily conserved cellular catabolic process $(8,28)$. To verify this pivotal phenomenon, we performed a GFP-LC3B puncta-formation assay and an LC3B conversion assay (29). Using GBM cells (T98G) that stably express a GFP-LC3B fusion protein, the localization of GFP-LC3B was examined by fluorescent microscopy. GFP-LC3B puncta that appear in the cytoplasm reflect the recruitment of LC3B proteins to autophagosomes. As shown in Fig. 3B, a significant increase in GFP-LC3B puncta was noted in hypoxic cells, and this result was confirmed by the quantification of GFP-LC3B dots per cell. Moreover, we detected the conversion of LC3B-I to LC3B-II and P62 (autophagy marker) expression together with CREBRF and CREB3 protein levels by western blot analysis under different hypoxia treatment times. Consistent with the GFP-LC3B puncta-formation assay, hypoxia led to a significant time-dependent upregulation of LC3B-II and 
A
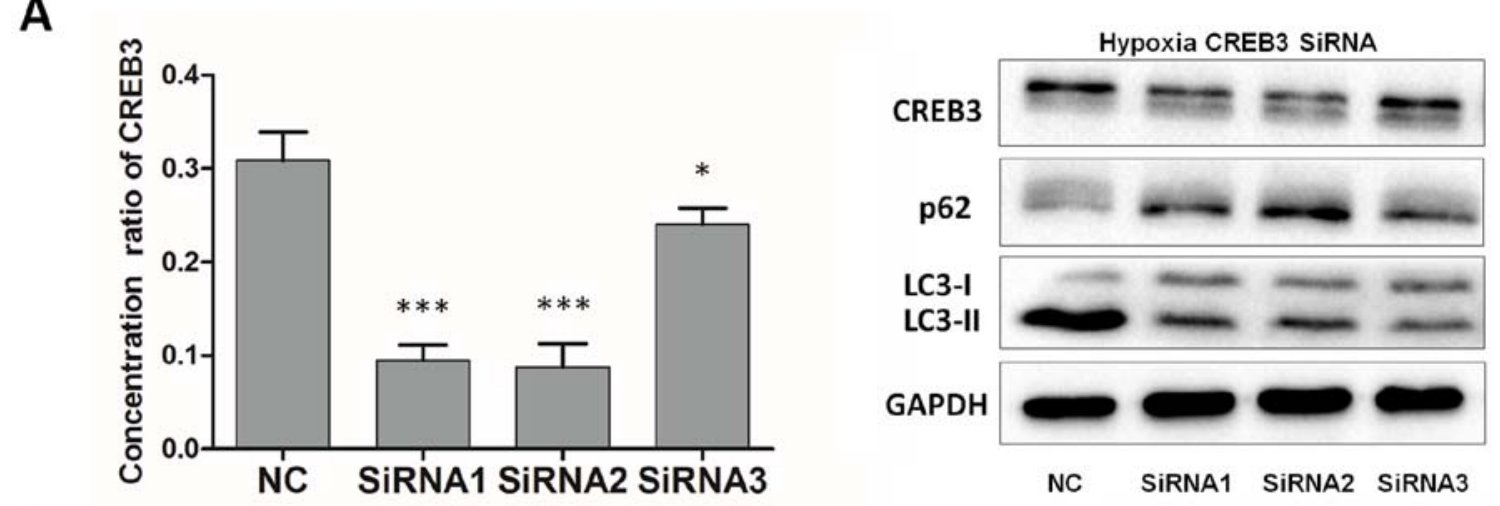

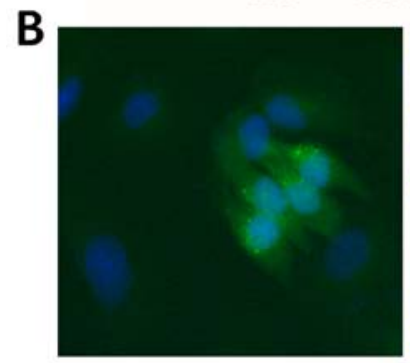

Normoxia

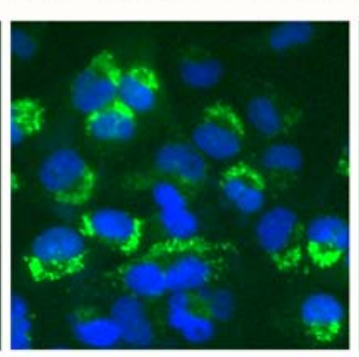

Hypoxia

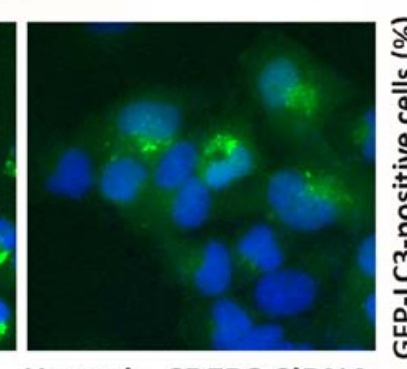

Hypoxia-CREB3 SiRNA

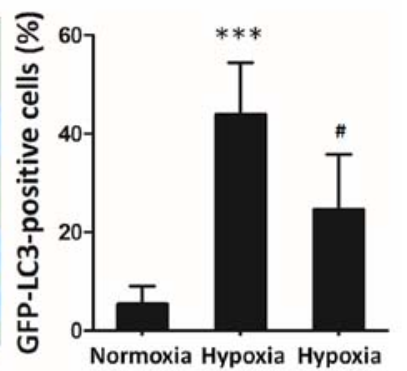

CREB3 SIRNA

Figure 4. Inhibition of endogenous CREB3 represses hypoxia-induced autophagy in glioblastoma cells. (A) CREB3 mRNA and protein levels in T98G cells transfected with CREB3 siRNAs and a negative control. At $72 \mathrm{~h}$ after transfection, total protein was extracted and analyzed by western blotting. GAPDH was used as the loading control. (B) CREB3 siRNA inhibits GFP-LC3B translocation. pSELECT-GFP-LC3B-transfected T98G cells treated with hypoxia for $24 \mathrm{~h}$. Quantitative analysis of GFP-LC3B puncta is presented in the right panel. The data presented are the mean $\pm \mathrm{SD}$ of three independent experiments. ${ }^{*} \mathrm{P}<0.05$, ${ }^{* * * *} \mathrm{P}<0.001$, two-tailed t-test.

downregulation of P62 (Fig. 3C). Also consistent with the quantitative real-time PCR results, hypoxia downregulated CREBRF and upregulated CREB3 protein levels in a timedependent manner (Fig. 3C). Thus, both assays suggested that hypoxia induces autophagosome accumulation and that CREBRF/CREB3 may be involved in this process.

Inhibition of endogenous CREB3 represses hypoxia-induced autophagy in glioblastoma cells. To further support the fact that endogenous CREB3 is involved in hypoxia-induced autophagy, we examined LC3B-II and P62 protein levels after attenuating CREB3 activity in hypoxic glioblastoma cells using RNA interference (RNAi) (Fig. 4A, left). Western blot analysis clearly indicated that CREB3 knockdown inhibited the hypoxia-induced LC3B-II increase and P62 degradation in glioblastoma cells (Fig. 4A, right). Using T98G cells transiently transfected with GFP-LC3B, our study revealed that the inhibition of endogenous CREB3 inhibited autophagy under hypoxic conditions (Fig. 4B). The data indicate that a blockade of endogenous CREB3 repressed autophagy in hypoxic glioblastoma cells.

CREB3 enhances hypoxia-induced autophagy by targeting ATG5 in human glioblastoma cells. To elucidate the mechanisms of CREB3-induced autophagy, several autophagy-related genes (ATGs) were examined by quantitative real-time PCR in T98G cells, revealing a significant decrease in ATG5 after CREB3 knockdown (Fig. 5A) that was confirmed by western blot analysis in both T98G and U87 cell lines (Fig. 5B). To further investigate the relationship between CREB3 and ATG5 in hypoxic glioblastoma cells, we collected 3 fresh, surgically removed WHO grade IV glioma tissue samples for fastfrozen sectioning, and staining revealed the co-localization of CREB3, ATG5, and LC3B proteins in high-grade glioma tissues (Fig. 5C).

CREB3 knockdown induces autophagy-enhanced apoptosis in GBM cells. In recent years, accumulating evidence has demonstrated that autophagy may serve as a protective mechanism in tumor cells and that therapy-induced apoptosis can be potentiated by autophagy inhibition $(30,31)$. To determine the biological significance of CREB3-induced autophagy on apoptotic cell death, CREB3 interfering RNA was utilized in glioblastoma cells. A CCK-8 assay was utilized to determine cell viability. TUNEL staining assays and Annexin V-FITC/ PI were performed to examine the level of apoptosis in glioblastoma cells. As shown in Fig. 6A, CREB3 knock-down significantly suppressed glioblastoma cells exclusively under hypoxic conditions. Consistent with this observation, hypoxiainduced apoptotic cell death was augmented in the presence of CREB3 interfering RNA, which was demonstrated by the TUNEL assay (Fig. 6B) and the Annexin V-FITC/PI assay (Fig. 6C). In the process of apoptosis, caspase 3 , as the final effector molecule, can be cleaved and activated as the most common characteristic of apoptosis. Subsequently, we examined cleaved caspase 3 protein levels by western blot analysis, and the data further indicated that CREB3 knock-down led to the activation of caspase 3 apoptotic signaling pathways (Fig. 6D). This type of crosstalk between autophagy and apoptosis provides a possible avenue for interference with 
A

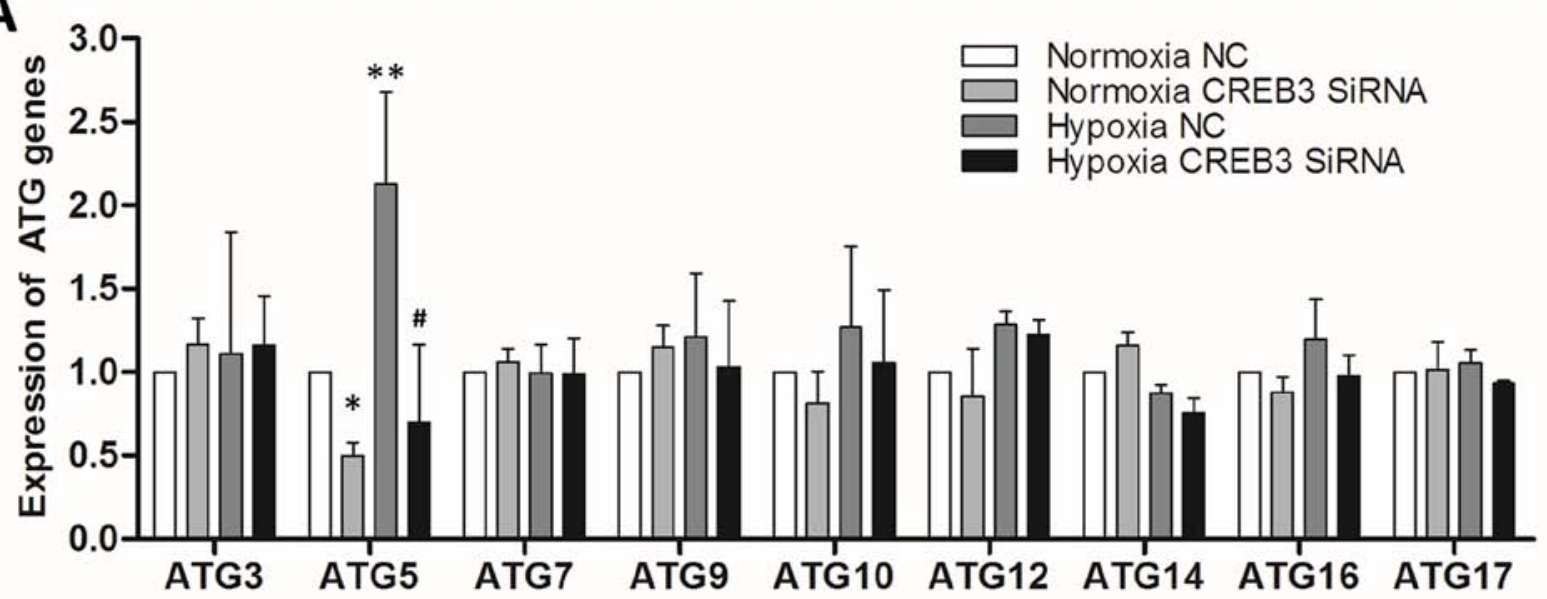

B

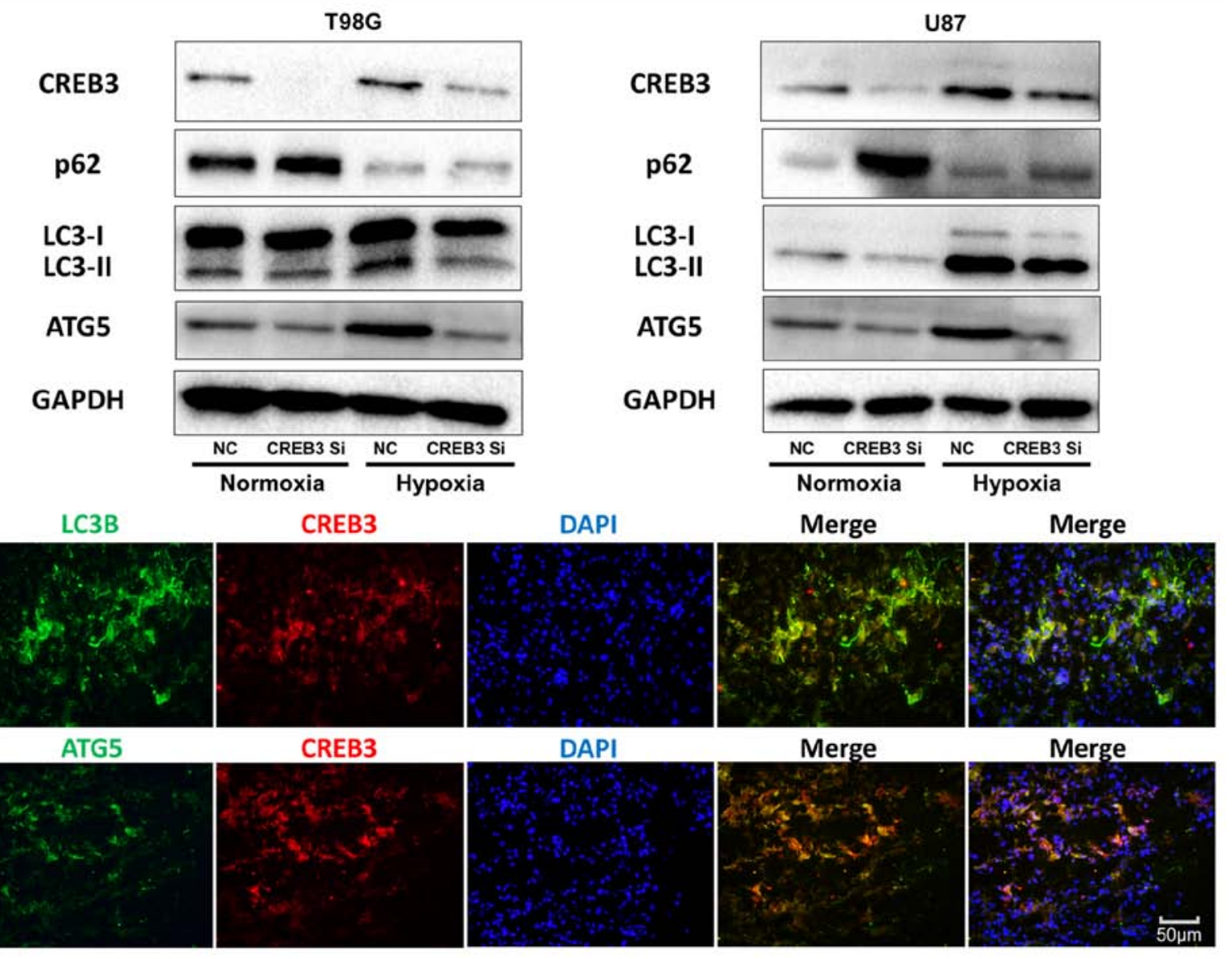

Figure 5. CREB3 enhances hypoxia-induced autophagy by directly targeting ATG5 in human glioblastoma cells. (A) CREB3-induced autophagy-related genes (ATGs) in T98G cells by quantitative real-time PCR. ${ }^{*} \mathrm{P}<0.05,{ }^{* *} \mathrm{P}<0.01$, two-tailed t-test. (B) CREB3 siRNA knockdown decreases autophagy levels and ATG5 protein levels in T98G and U87 cells. (C) Co-localization of LC3B, ATG5 and CREB3 in a sample of WHO grade IV glioma tissues. Three surgically removed, fresh WHO grade IV glioma tissues were subject to fast-frozen sectioning and staining.

hypoxia-induced autophagy of glioblastoma cells through anti-CREB3 adjuvant therapy to treat glioma patients.

\section{Discussion}

Autophagy benefits glioblastoma cell survival under hypoxic metabolic stress and mediates resistance to radiotherapy and chemotherapy $(32,33)$, and these strategies trigger cellular autophagy (14). Recently, accumulating evidence has demon- strated that the inhibition of autophagy triggers apoptosis and kills more tumor cells (30). Our study also revealed a novel anti-autophagy therapeutic target by regulating hypoxiadependent CREBRF/CREB3 in glioblastoma cells. Given that upregulated autophagy inhibits tumor apoptosis and promotes proliferation (34), we inhibited the CREB3-dependent pathway using specific siRNAs that significantly suppressed glioblastoma cell viability by converting autophagy into apoptosis. This work is significant because it suggests a potential 
A

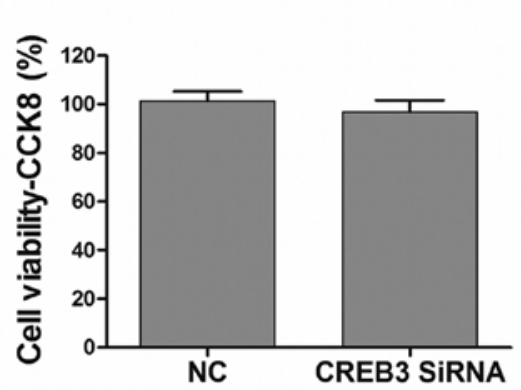

B

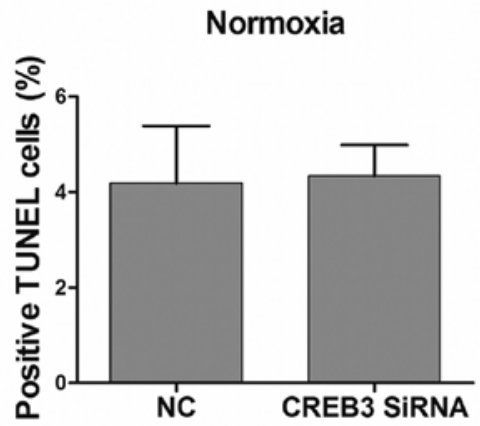

C

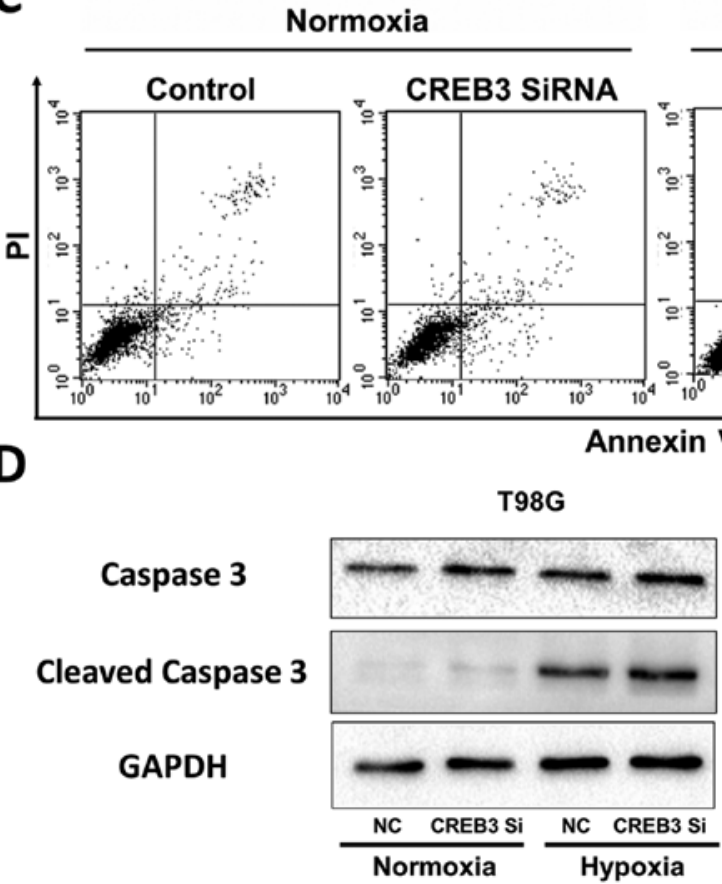

Hypoxia

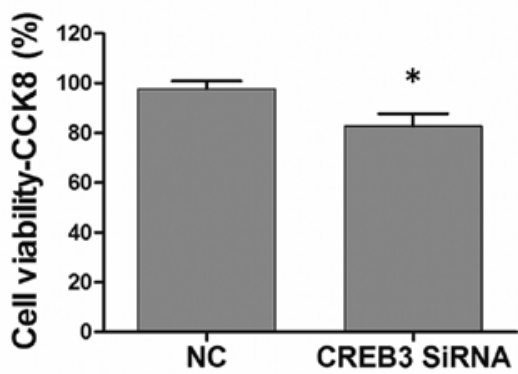

Hypoxia

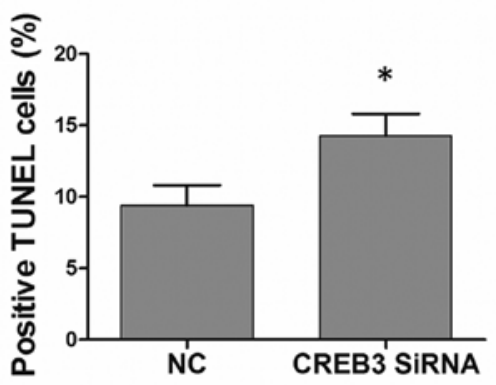

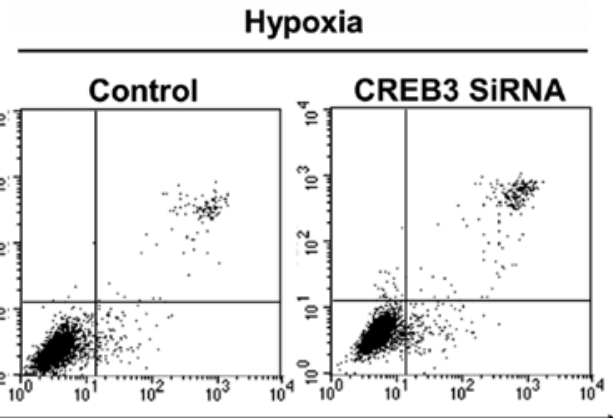

U87

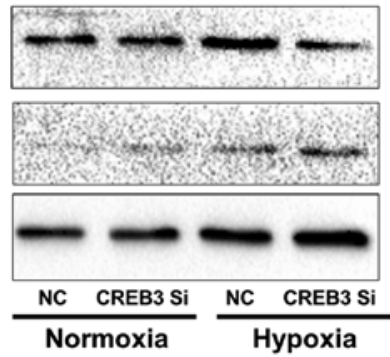

Figure 6. CREB3 knockdown induces autophagy-enhanced apoptosis in GBM cells. (A) A CCK-8 assay was performed to assess cell viability in hypoxiatreated T98G cells after transfection for $48 \mathrm{~h}$. (B and C) TUNEL staining and Annexin V-FITC/PI assays were performed to examine the level of apoptosis in hypoxic glioblastoma cells after transfection for $48 \mathrm{~h}$. (D) Cleavage of caspase 3 was induced by CREB3 siRNA transfection in hypoxic glioblastoma cells. Caspase- 3 and cleaved caspase 3 levels were examined by western blot analysis. GAPDH served as the loading control. The data are the mean \pm SD. ${ }^{*} \mathrm{P}<0.05$ compared with the control group.

anti-glioma effect of the anti-CREB3 strategy in vitro for the first time and characterizes the pivotal role of the CREBRF/ CREB3/ATG5 pathway in hypoxia-induced autophagy in glioblastoma cells.

The tumor microenvironment plays a critical role in tumor progression. As the tumor rapidly outgrows its blood supply, malignant proliferative tumor cells are deprived of oxygen (35). Tumor hypoxia can powerfully induce cells to develop an aggressive and treatment-resistant phenotype that leads to rapid progression and poor prognosis $(6,13)$, this development is even the case for immune cells (36-39). HIF-1 $\alpha$ is a key transcription factor in the adaptation to hypoxic stress and plays an important role in hypoxia-induced autophagy (40), which activates several autophagy-inducing molecules, such as BNIP3 (41) and IGFBP3 (42). In addition, HIF-1 $\alpha$-independent AMPK activates more severe hypoxia, and AMPK could contribute to autophagy via the mTOR inhibition mechanism $(43,44)$. Nevertheless, in this study, we found that CREB3 also 
plays a role in hypoxia-induced autophagy. We also assumed that CREBRF might act as a tumor suppressor by inhibiting CREB3-induced autophagy. In addition, CREBRF and CREB3 levels in patient glioma samples might also be used as prognostic factors.

CREBRF is a negative regulator of CREB3 that can recruit nuclear CREB3 to discrete foci in the nucleus, promote CREB3 protein degradation and repress CREB3-mediated activation of unfolded protein response element (UPRE)containing promoters (22). CREB3 (also called Luman or LZIP) is the primary member of the CREB3 family. All CREB3 family members appear to play a role in the unfolded protein response (UPR) (45), during which endoplasmic reticulum (ER)-resident molecular chaperones and foldases are induced, attenuating translation to reduce the load on the ER (46). Unfolded proteins can also be targeted for proteasomal degradation via ubiquitination (47). CREB (cyclic-AMPresponsive element binding) family proteins, including CREB1, CREB2 and CREB3, bind to the cAMP-responsive element (CRE) recognition sequence of cAMP-sensitive genes to regulate transcription (23). CREB1 upregulates autophagy genes ATG7, Ulk1, and Tfeb (25) and CREBRF is involved in inducing cell apoptosis through the ER stress pathway (27). Our study is the first to confirm that CREB3 is also involved in regulating autophagy in tumor cells.

The cAMP signal transduction pathway is activated through ligand binding to G-protein coupled receptors and culminates with the phosphorylation of the CREB family protein, thus allowing it to bind the cAMP responsive element (CRE) recognition sequence of cAMP-sensitive genes and to regulate transcription (23). Details regarding the positive correlation between CREB3 and ATG5 in our study remain unclear. The CRE sequence in ATG5 must be verified first. Alternatively, other intermediate regulator molecules might play a vital role if the regulation of CREB3 is indirect.

In conclusion, this study demonstrated that the hypoxiadependent CREBRF/CREB3 pathway is a potent autophagy regulator in glioma by regulating ATG5 and that CREB3induced autophagy protects glioblastoma cells from apoptotic death. We also suggested the potential uses for anti-CREB3 therapeutic strategies in adjuvant therapy for glioma patients and the prognosis predicting effect of CREBRF in tissues of glioma patients. Future in vitro studies should be directed toward the identification of the regulation mechanism of CREB3 and ATG5, and future in vivo research should be investigated.

\section{Acknowledgements}

We thank Professor Xun Qu for helpful comments and advice on this study, and D. Nicole, Senior Editor at American Journal Experts, for language advice. This study was supported by grants from the National Natural Science Foundation of China (nos. 81101594, 81372719, 81172403, 81300510, 81402077, 81571284 and 91542115) and Taishan Scholars of Shandong Province of China (no. ts201511093).

\section{References}

1. Ohgaki H: Epidemiology of brain tumors. Methods Mol Biol 472: 323-342, 2009 .
2. Ostrom QT, Gittleman H, Stetson L, Virk SM and BarnholtzSloan JS: Epidemiology of gliomas. Cancer Treat Res 163: 1-14, 2015.

3. Davis ME and Stoiber AM: Glioblastoma multiforme: Enhancing survival and quality of life. Clin J Oncol Nurs 15: 291-297, 2011.

4. Sayegh ET, Kaur G, Bloch O and Parsa AT: Systematic review of protein biomarkers of invasive behavior in glioblastoma. Mol Neurobiol 49: 1212-1244, 2014.

5. McNamara MG and Mason WP: Antiangiogenic therapies in glioblastoma multiforme. Expert Rev Anticancer Ther 12: 643-654, 2012.

6. Vaupel P: Hypoxia and aggressive tumor phenotype: Implications for therapy and prognosis. Oncologist 13 (Suppl 3): 21-26, 2008.

7. Mongiardi MP: Angiogenesis and hypoxia in glioblastoma: A focus on cancer stem cells. CNS Neurol Disord Drug Targets 11: 878-883, 2012

8. Mazure NM and Pouysségur J: Hypoxia-induced autophagy: Cell death or cell survival? Curr Opin Cell Biol 22: 177-180, 2010.

9. Yuan G, Yan SF, Xue H, Zhang P, Sun JT and Li G: Cucurbitacin I induces protective autophagy in glioblastoma in vitro and in vivo. J Biol Chem 289: 10607-10619, 2014.

10. Harris AL: Hypoxia - a key regulatory factor in tumour growth. Nat Rev Cancer 2: 38-47, 2002.

11. Evans SM, Judy KD, Dunphy I, Jenkins WT, Hwang WT, Nelson PT, Lustig RA, Jenkins K, Magarelli DP, Hahn SM, et al: Hypoxia is important in the biology and aggression of human glial brain tumors. Clin Cancer Res 10: 8177-8184, 2004.

12. Brahimi-Horn $\mathrm{C}$ and Pouysségur J: The role of the hypoxiainducible factor in tumor metabolism growth and invasion. Bull Cancer 93: E73-E80, 2006.

13. Ji RC: Hypoxia and lymphangiogenesis in tumor microenvironment and metastasis. Cancer Lett 346: 6-16, 2014.

14. Hu YL, Jahangiri A, De Lay M and Aghi MK: Hypoxia-induced tumor cell autophagy mediates resistance to anti-angiogenic therapy. Autophagy 8: 979-981, 2012.

15. Meijer AJ and Codogno P: Autophagy: Regulation and role in disease. Crit Rev Clin Lab Sci 46: 210-240, 2009.

16. Chen $\mathrm{N}$ and Karantza-Wadsworth V: Role and regulation of autophagy in cancer. Biochim Biophys Acta 1793: 1516-1523, 2009.

17. Shintani T and Klionsky DJ: Autophagy in health and disease: A double-edged sword. Science 306: 990-995, 2004.

18. Thorburn A, Thamm DH and Gustafson DL: Autophagy and cancer therapy. Mol Pharmacol 85: 830-838, 2014.

19. Gewirtz DA: The four faces of autophagy: Implications for cancer therapy. Cancer Res 74: 647-651, 2014.

20. Guo JY, Xia B and White E: Autophagy-mediated tumor promotion. Cell 155: 1216-1219, 2013.

21. Aredia F, Guamán Ortiz LM, Giansanti V and Scovassi AI: Autophagy and cancer. Cells 1: 520-534, 2012.

22. Audas TE, Li Y, Liang G and Lu R: A novel protein, Luman/ CREB3 recruitment factor, inhibits Luman activation of the unfolded protein response. Mol Cell Biol 28: 3952-3966, 2008.

23. Mamdani F, Alda M, Grof P, Young LT, Rouleau G and Turecki G: Lithium response and genetic variation in the CREB family of genes. Am J Med Genet B Neuropsychiatr Genet 147B: 500-504, 2008.

24. Cao G, Ni X, Jiang M, Ma Y, Cheng H, Guo L, Ji C, Gu S, Xie Y and Mao Y: Molecular cloning and characterization of a novel human cAMP response element-binding (CREB) gene (CREB4). J Hum Genet 47: 373-376, 2002.

25. Seok S, Fu T, Choi SE, Li Y, Zhu R, Kumar S, Sun X, Yoon G, Kang Y, Zhong W, et al: Transcriptional regulation of autophagy by an FXR-CREB axis. Nature 516: 108-111, 2014.

26. Liu YL, Lai F, Wilmott JS, Yan XG, Liu XY, Luan Q, Guo ST, Jiang CC, Tseng HY, Scolyer RA, et al: Noxa upregulation by oncogenic activation of MEK/ERK through CREB promotes autophagy in human melanoma cells. Oncotarget 5: 11237-11251, 2014.

27. Yang Y, Lin P, Chen F, Wang A, Lan X, Song Y and Jin Y: Luman recruiting factor regulates endoplasmic reticulum stress in mouse ovarian granulosa cell apoptosis. Theriogenology 79: 633-639. e1-3, 2013.

28. Mucaj V, Shay JE and Simon MC: Effects of hypoxia and HIFs on cancer metabolism. Int J Hematol 95: 464-470, 2012.

29. Mizushima N and Yoshimori T: How to interpret LC3 immunoblotting. Autophagy 3: 542-545, 2007.

30. Boya P, González-Polo RA, Casares N, Perfettini JL, Dessen P, Larochette N, Métivier D, Meley D, Souquere S, Yoshimori T, et al: Inhibition of macroautophagy triggers apoptosis. Mol Cell Biol 25: 1025-1040, 2005 
31. Wu H, Che X, Zheng Q, Wu A, Pan K, Shao A, Wu Q, Zhang J and Hong Y: Caspases: A molecular switch node in the crosstalk between autophagy and apoptosis. Int J Biol Sci 10: 1072-1083, 2014.

32. Yang Z and Klionsky DJ: Eaten alive: A history of macroautophagy. Nat Cell Biol 12: 814-822, 2010.

33. Marino ML, Pellegrini P, Di Lernia G, Djavaheri-Mergny M, Brnjic S, Zhang X, Hägg M, Linder S, Fais S, Codogno P, et al: Autophagy is a protective mechanism for human melanoma cells under acidic stress. J Biol Chem 287: 30664-30676, 2012.

34. Mukhopadhyay S, Panda PK, Sinha N, Das DN and Bhutia SK: Autophagy and apoptosis: Where do they meet? Apoptosis 19: 555-566, 2014.

35. Eng JW, Kokolus KM, Reed CB, Hylander BL, Ma WW and Repasky EA: A nervous tumor microenvironment: The impact of adrenergic stress on cancer cells, immunosuppression, and immunotherapeutic response. Cancer Immunol Immunother 63: $1115-1128,2014$

36. Yang M, Ma C, Liu S, Shao Q, Gao W, Song B, Sun J, Xie Q, Zhang Y, Feng A, et al: HIF-dependent induction of adenosine receptor $\mathrm{A} 2 \mathrm{~b}$ skews human dendritic cells to a Th2-stimulating phenotype under hypoxia. Immunol Cell Biol 88: 165-171, 2010.

37. Seo YJ, Koh SH, Kang HJ, Shin HY, Jeong G and Ahn HS: Hypoxia inhibits the SDF-1-dependent migration of human leukemic cell line HL-60 via blocking of Akt activation. Biochem Biophys Res Commun 364: 388-394, 2007.

38. Qu X, Yang MX, Kong BH, Qi L, Lam QL, Yan S, Li P, Zhang M and Lu L: Hypoxia inhibits the migratory capacity of human monocyte-derived dendritic cells. Immunol Cell Biol 83: 668-673, 2005

39. Zhao W, Darmanin S, Fu Q, Chen J, Cui H, Wang J, Okada F, Hamada J, Hattori Y, Kondo T, et al: Hypoxia suppresses the production of matrix metalloproteinases and the migration of human monocyte-derived dendritic cells. Eur J Immunol 35 : 3468-3477, 2005.
40. Unwith S, Zhao H, Hennah L and Ma D: The potential role of HIF on tumour progression and dissemination. Int J Cancer 136: 2491-2503, 2015.

41. Hu YL, DeLay M, Jahangiri A, Molinaro AM, Rose SD, Carbonell WS and Aghi MK: Hypoxia-induced autophagy promotes tumor cell survival and adaptation to antiangiogenic treatment in glioblastoma. Cancer Res 72: 1773-1783, 2012.

42. Hsieh DJ, Kuo WW, Lai YP, Shibu MA, Shen CY, Pai P, Yeh YL, Lin JY, Viswanadha VP and Huang CY: $17 \beta$-estradiol and/ or estrogen receptor $\beta$ attenuate the autophagic and apoptotic effects induced by prolonged hypoxia through HIF-1 $\alpha$-mediated BNIP3 and IGFBP-3 signaling blockage. Cell Physiol Biochem 36: 274-284, 2015.

43. Gwinn DM, Shackelford DB, Egan DF, Mihaylova MM, Mery A, Vasquez DS, Turk BE and Shaw RJ: AMPK phosphorylation of raptor mediates a metabolic checkpoint. Mol Cell 30: 214-226, 2008.

44. Jung CH, Jun CB, Ro SH, Kim YM, Otto NM, Cao J, Kundu M and Kim DH: ULK-Atg13-FIP200 complexes mediate mTOR signaling to the autophagy machinery. Mol Biol Cell 20: 1992-2003, 2009.

45. Lu R, Yang P, O'Hare P and Misra V: Luman, a new member of the CREB/ATF family, binds to herpes simplex virus VP16associated host cellular factor. Mol Cell Biol 17: 5117-5126, 1997.

46. Mori K: Tripartite management of unfolded proteins in the endoplasmic reticulum. Cell 101: 451-454, 2000.

47. Sifers RN: Cell biology. protein degradation unlocked. Science 299: 1330-1331, 2003. 